\title{
The research on the consistency of the testing accuracy for smoke measuring equipment based on ionization chamber principle
}

\author{
Ting Ting WANG ${ }^{1, a}$, Pei ZHANG ${ }^{1}$, Hong ZHANG ${ }^{1}$, Lei Lei $\mathrm{XIE}^{1}$, Ya Jun YAN ${ }^{1}$, Miao Miao DAI ${ }^{1}$, and Ya Yun WANG ${ }^{1}$ \\ ${ }^{1}$ Shanghai Institute of Quality Inspection and Technical Research, 201114 Shanghai, China
}

\begin{abstract}
A convenient method is proposed here to determine the relative accuracy of measuring ionization chamber (MIC). A non-dimensional quantity is presented here to characterize the relative accuracy of the testing sample to the reference sample. There are two key points of the program. One is comparison by pairs. The other is eliminating the uncertainty in the program by exchanging the position and control unit in each testing group.
\end{abstract}

\section{Introduction}

As we known, there are two kinds of widely used smoke measuring equipment, which are obscuration meter and measuring ionization chamber (MIC) [1]. Smoke density can be measured by different techniques [2-6]. However, for reference measurements of smoke density in connection with testing of ionization smoke detectors for automatic fire detection systems, it is advantageous to use a measuring instrument based on the ionization chamber principle. This is because of the complex nature of smoke, which means that measuring errors are likely to occur if the reference measuring instrument reacts to other properties of the smoke than the detector under test.

When the measuring ionization chamber (MIC) [79] is used in the smoke tunnel measurements and fire sensitivity tests, the high measuring accuracy is very important for the overall measuring accuracy of the whole system is limited by the measuring accuracy of the measuring ionization chamber (MIC). The radioactive source in the MIC makes the certification of MIC very expensive and complicated. A convenient method is proposed here to determine the relative accuracy of measuring ionization chamber (MIC) which may be widely used by testing laboratory and manufactory.

\section{Measuring ionization chamber (MIC)}

The response threshold of detectors using ionization is characterized by a non-dimensional quantity $\mathrm{y}$ or $\mathrm{X}$ which are derived from the relative change of the current flowing in a measuring ionization chamber, and which are related to the particle concentration of the test aerosol, measured in the proximity of the detector, at the moment that it generates an alarm signal.
According to BS EN54-7:2001 "Fire detection and fire alarm systems" and other relative standard, MIC consists of a measuring chamber, an electronic amplifier and a method of continuously sucking in a sample of the aerosol or smoke to be measured.

The measuring chamber contains a measuring volume a suitable means by which the sampled air is sucked in and passes the measuring volume in such a way that the aerosol/smoke particles diffuse into the volume. This diffusion is such that the flow of ions within the measuring volume is not disturbed by air movements. The air within the measuring volume is ionized by alpha radiation from an americium radioactive source, such that there is bipolar flow of ions when an electrical voltage is applied between the electrodes. This flow of ions is affected by the aerosol or smoke particles in a known manner. The relative variation in the current of ions is used as a measurement of the aerosol or smoke concentration.

The measured smoke densities is expressed in terms of the dimensionless quantity defined by the equation:

$$
\mathrm{X}=\frac{\mathrm{I}_{0}-\mathrm{I}}{\mathrm{I}_{0}}, \quad 0 \leq \mathrm{X} \leq 1
$$

Where $I_{0}$ is the quiescent ionization chamber current in clean air and $\mathrm{I}$ is the ionization chamber current in presence of smoke.

\section{Testing program}

\subsection{Basis of the program}

According to the chapter 4.4 of CNAS-CL 06:2014 "The traceability requirements of the measured results", a program is designed to get the relative accuracy of the measuring ionization chamber (MIC). According to

\footnotetext{
${ }^{\mathrm{a}}$ Corresponding author: wangtt@sqi.org.cn
} 
CNAS-CL 06:2014, several methods of measurement traceability have been pointed.

When measuring results can not be traced back to the international system of units (SI) or is not related to SI, the measurement should be traced back to the RM, method/standard agreed by conventional measurement angecy, or through the way such as comparison between laboratory, to prove the consistency of the measurement result with other laboratories.

\subsection{Key points of the program}

A non-dimensional quantity $\mathrm{R}_{\mathrm{ns}}$ is used here to characterize the relative accuracy of the test sample named $\mathrm{n}$ and reference sample named $\mathrm{S}$.

There are two key points of the program. One is comparison by pairs which is tested as a group. The other is eliminating the uncertainty in the program as much as possible.

\subsubsection{Comparison by pairs}

A specified measuring ionization chamber (MIC) is chosen as reference sample S. Other testing measuring ionization chamber (MIC) are marked as No. 1, 2,...N. Compare the testing sample $\mathrm{n}$ and the reference sample $\mathrm{S}$ respectively. Take a testing sample named $n$ and place it with the reference sample $S$ in the same smoke environment. Test the non-dimensional quantity $\mathrm{X}$ of both samples and compare them. If you have many testing samples, you should make the testing pair by pair. Schematic diagram is shown in figure 1.

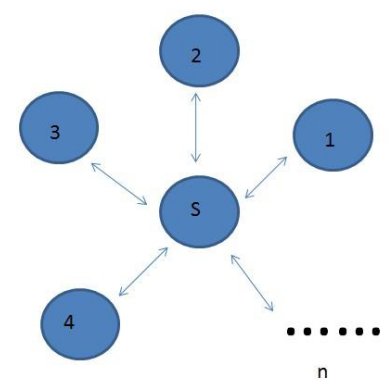

Figure 1. Schematic diagram of Comparison by pairs.

\subsubsection{Eliminating the uncertainty}

There are two factors that affect the measurement uncertainty, which are the position of the samples and the selection of the control unit.

\section{Position of the samples:}

Sample $\mathrm{n}$ and $\mathrm{S}$ are placed in the vertical direction of the smoke flow. In order to fully eliminate the effects of inhomogeneous smoke in the tunnel, the testing must be carried out twice when comparing the sample $\mathrm{n}$ and $\mathrm{S}$ by exchanging the position. Swap positions can effectively reduce the measurement uncertainty. Schematic diagram is shown in figure 2 .

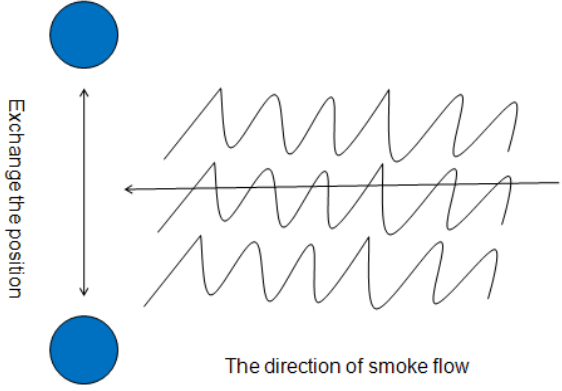

Figure 2. Schematic diagram of the position of the samples.

\section{Selecting the measuring ionization chamber (MIC) control unit:}

For the testing must be done pair by pair, two measuring ionization chamber (MIC) control units are needed here. In order to eliminate the measurement uncertainty, the same type measuring ionization chamber (MIC) control units come from the same manufacturer should be selected. Also, swap the control unit can eliminate the tiny difference between the control units.

\subsection{Testing procedures}

Here is the detail procedure:

1. Clean and alignment the smoke tunnel and measuring ionization chambers (MICs) to ensure they work property.

2. Install the samples in the correct position and connect the control units respectively.

3. Preheat the equipment for 15 minutes.

4. Check the performance of the measuring ionization chamber (MIC). Adjust the chamber voltage VDC.

5. Adjust the aerosol generator. The measuring ionization chambers (MICs) (sample $\mathrm{n}$ and S) are exposed to a slowly increasing smoke density produced by a paraffin oil aerosol generator. The rate of increase of aerosol density is $0.05 \mathrm{~min}^{-1}$. The flow rate of aerosol is $201 \mathrm{~min}^{-1}$.

6. Record the values $\mathrm{X}$ of the sample $\mathrm{n}$ and $\mathrm{S}$. Record as Xn and Xs.

7. Denote Xs and Xn abscissa and ordinate respectively. Carry on the linear numerical fitting to get the slope $\mathrm{R}_{\mathrm{ns}-1}$.

8. Swap the position and the control units of sample $n$ and $\mathrm{S}$ and record the values $\mathrm{X}$ also.

9. Repeat the step 7 to get the slope $R_{n s-2}, R_{n s-3}, R_{n s-4}$.

10. The relative accuracy of sample $n$ is $R_{n s}$, which is $\left(R_{n s-1}+R_{n s-2}+R_{n s-3}+R_{n s-4}\right) / 4$.

11. The relative error of the sample $n$ is $\left|1-R_{n s}\right| \times 100 \%$. If the relative error is less than $5 \%$, the relative accuracy of the testing sample $\mathrm{n}$ to the relative $\mathrm{S}$ is good. This tolerance $5 \%$ comes from chapter 5.1 .4 of BS EN54-7:2001.

Note: Must empty the smoke tunnel before each testing. 


\section{Example}

Here, an example is presented to testify our program.

Testing environment: Air temperature is $20.6{ }^{\circ} \mathrm{C}$ and air humidity is $45.4 \%$. Air velocity is $0.2 \mathrm{~m} / \mathrm{s}$.

Condition: The rate of increase of aerosol density is $0.05 \mathrm{~min}^{-1}$. The flow rate of aerosol is $201 \mathrm{~min}^{-1}$. The flow rate of vacuum pump is $301 \mathrm{~min}^{-1}$.

Test equipment: measuring ionization chamber (MIC) : Delta MIC EC-912, smoke tunnel: AWT 2800.

Mark the testing sample as a. Based on the choice of location and control unit of the reference sample $\mathrm{S}$, There is four situations of each testing group.

\section{Situation 1:}

Table 1.The choice of location and control unit for situation 1.

\begin{tabular}{|l|l|}
\hline Location1, control unit 1 & $\bullet$ \\
\hline Location1, control unit 2 & \\
\hline Location2, control unit 1 & \\
\hline Location2, control unit 2 & \\
\hline
\end{tabular}

Table 2 shows the values for under testing sample a and reference sample $\mathrm{S}$ in situation 1. Figure 3 shows the values $X$ of sample a to $S$.

Table 2. Values for under testing sample a and reference sample $\mathrm{S}$ in situation 1 .

\begin{tabular}{|c|c|}
\hline Xa (VDC:18.8V) & Xs (VDC: 19.2V) \\
\hline 0.00 & 0.00 \\
\hline 0.05 & 0.05 \\
\hline 0.11 & 0.10 \\
\hline 0.16 & 0.15 \\
\hline 0.20 & 0.20 \\
\hline 0.26 & 0.25 \\
\hline 0.32 & 0.30 \\
\hline 0.36 & 0.35 \\
\hline 0.41 & 0.40 \\
\hline 0.46 & 0.45 \\
\hline 0.50 & 0.50 \\
\hline
\end{tabular}

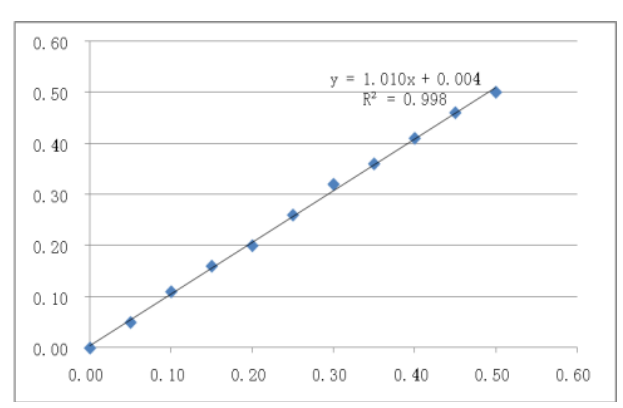

Figure 3. Values $\mathrm{X}$ of sample a to $\mathrm{S}$.

From figure 3 , it can be derived that the slope $R_{n s-1}$ is 1.010 , and the correlation coefficient is 0.998 .

\section{Situation 2:}

Table 3.The choice of location and control unit for situation 2 .

\begin{tabular}{|l|l|}
\hline Location1, control unit 2 & $\bullet$ \\
\hline Location2, control unit 1 & \\
\hline Location2, control unit 2 & \\
\hline
\end{tabular}

Table 4 shows the values for under testing sample a and reference sample $\mathrm{S}$ in situation 2. Figure 4 shows the values $\mathrm{X}$ of sample a to $\mathrm{S}$.

Table 4. Values for under testing sample a and reference sample $\mathrm{S}$ in situation 2 .

\begin{tabular}{|c|c|}
\hline Xa (VDC: 18.4V) & Xs (VDC:18.9V) \\
\hline 0.00 & 0.00 \\
\hline 0.05 & 0.06 \\
\hline 0.10 & 0.09 \\
\hline 0.15 & 0.15 \\
\hline 0.20 & 0.20 \\
\hline 0.25 & 0.25 \\
\hline 0.30 & 0.30 \\
\hline 0.35 & 0.35 \\
\hline 0.40 & 0.39 \\
\hline 0.45 & 0.44 \\
\hline 0.50 & 0.49 \\
\hline
\end{tabular}

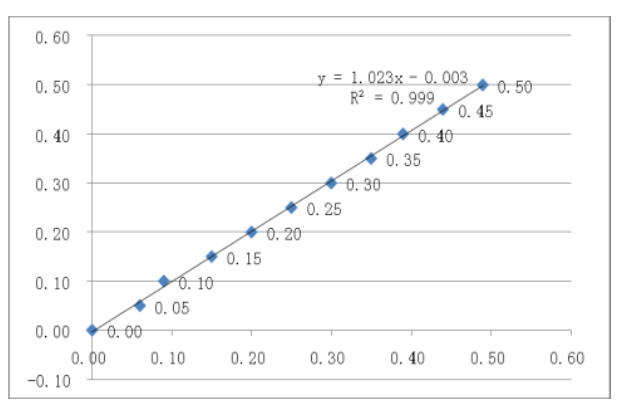

Figure 4. Values $\mathrm{X}$ of sample a to $\mathrm{S}$.

From figure 4 , it can be derived that the slope $\mathrm{R}_{\mathrm{ns}-2}$ is 1.023 , and the correlation coefficient is 0.999 .

\section{Situation 3:}

Table 5. The choice of location and control unit for situation 3 .

\begin{tabular}{|l|l|}
\hline Location1, control unit 1 & \\
\hline Location1, control unit 2 & \\
\hline Location2, control unit 1 & $\bullet$ \\
\hline Location2, control unit 2 & \\
\hline
\end{tabular}

Table 6 shows the values for under testing sample a and reference sample $\mathrm{S}$ in situation 3. Figure 5 shows the values $X$ of sample a to $S$.

Table 6. Values for under testing sample a and reference sample $\mathrm{S}$ for situation 3 .

\begin{tabular}{|c|c|}
\hline Xa (VDC:18.8V) & Xs (VDC:19.4V) \\
\hline 0.00 & 0.00 \\
\hline 0.05 & 0.06 \\
\hline 0.10 & 0.11 \\
\hline 0.15 & 0.16 \\
\hline 0.20 & 0.21 \\
\hline 0.25 & 0.25 \\
\hline 0.30 & 0.30 \\
\hline
\end{tabular}




\begin{tabular}{|l|l|}
\hline 0.35 & 0.36 \\
\hline 0.40 & 0.41 \\
\hline 0.45 & 0.46 \\
\hline 0.50 & 0.50 \\
\hline
\end{tabular}

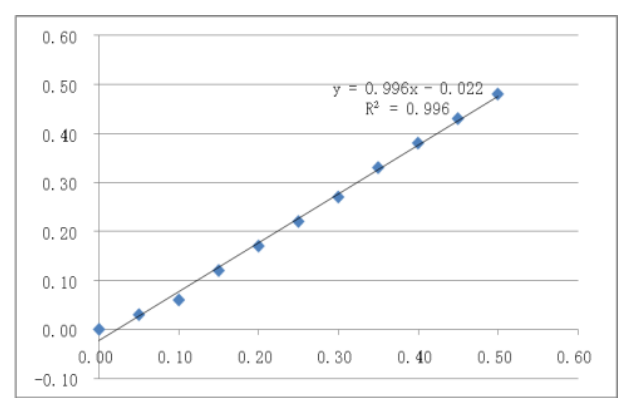

Figure 5. Values $\mathrm{X}$ of sample a to $\mathrm{S}$.

From figure 5 , it can be derived that the slope $\mathrm{R}_{\mathrm{ns}-3}$ is 0.996 , and the correlation coefficient is 0.996 .

\section{Situation 4:}

Table 7.The choice of location and control unit for situation 4 .

\begin{tabular}{|l|c|}
\hline Location1, control unit 1 & \\
\hline Location1, control unit 2 & \\
\hline Location2, control unit 1 & \\
\hline Location2, control unit 2 & $\bullet$ \\
\hline
\end{tabular}

Table 8 shows the values for under testing sample a and reference sample $S$ in situation 4. Figure 6 shows the values $X$ of sample a to $S$.

Table 8. Values for under testing sample a and reference sample $\mathrm{S}$ in situation 4 .

\begin{tabular}{|c|c|}
\hline Xa (VDC:19.0V) & Xs (VDC:19.8V) \\
\hline 0.00 & 0.00 \\
\hline 0.05 & 0.06 \\
\hline 0.10 & 0.11 \\
\hline 0.15 & 0.16 \\
\hline 0.20 & 0.21 \\
\hline 0.25 & 0.25 \\
\hline 0.30 & 0.30 \\
\hline 0.35 & 0.36 \\
\hline 0.40 & 0.41 \\
\hline 0.45 & 0.46 \\
\hline 0.50 & 0.50 \\
\hline
\end{tabular}

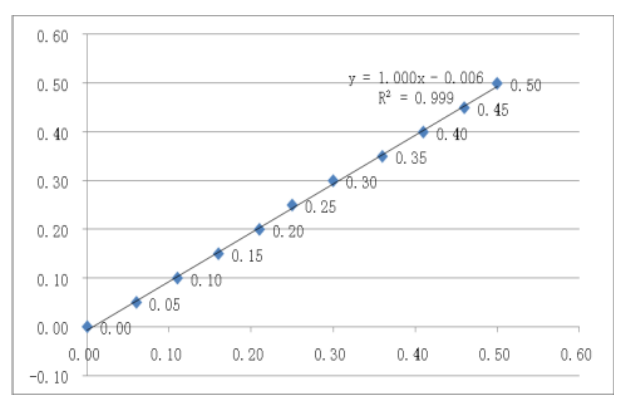

Figure 6. Values $\mathrm{X}$ of sample a to $\mathrm{S}$.
From figure 6 , it can be derived that the slope $\mathrm{R}_{\mathrm{ns}-4}$ is 1.000 , and the correlation coefficient is 0.999 .

Then the relative accuracy $R_{n s}$ of sample a to sample $\mathrm{S}$ is 1.00725 and the relative error of the sample a to sample $\mathrm{S}$ is $0.725 \%$. The relative error is smaller than the tolerance. So the testing performance of sample a and $\mathrm{s}$ has no obvious difference and they have a good consistency.

\section{Conclusion}

A convenient method is proposed in this paper to determine the relative accuracy of measuring ionization chamber (MIC). A non-dimensional quantity $\mathrm{R}_{\mathrm{ns}}$ is used here to characterize the relative accuracy of the testing sample named $\mathrm{n}$ and reference sample named $\mathrm{S}$. There are two key points of the program. One is comparison by pairs. The other is eliminating the uncertainty in the program by exchanging the position and control unit in each testing group. This method may be widely used by testing laboratory and manufactory.

This paper is funded by the project " $14 \mathrm{DZ} 2294100$ The platform construction project of professional testing technical service for digital electronic product" by shanghai science and technology committee.

\section{References}

1. S. Aggarwal, V. Motevalf, Fire safety J. 29, 99(1997).

2. S. J. Chen, D. C. Hovde, K. A. Peterson, A. W. Marshall, Fire safety J. 42, 507(2007).

3. M. A. Jackson, I. Robins, Fire safety J. 22, 185(1994).

4. S. Shibata, T. Higashino, A. Sawada, T. Oyabu, Y. Takei, H. Nabto, K. Toko, IEEJ Trans. on Sensors and Micromachines 130, 38(2010).

5. W. Krüll, R. Tobera, I. Willms, H. Essen, N. Wahl, Procedia Engin. 45, 584(2012).

6. G. Heskeatad, J. S. Newman, Fire safety J. 18, 355(1992).

7. N. G. Bernigau, H. O. Luck, J. Aerosol Sci. 17, 511(1986).

8. A. Scheidweiler, Fire Tech. 12, 113(1976).

9. C. Helsper, H. Fissan, J. Muggli, A. Scheidweiler, Fire Tech. 19, 14(1983). 